\title{
Fatigue, CTCAE 5.0
}

National Cancer Institute

\section{Source}

National Cancer Institute. Fatigue, CTCAE 5.0. NCI Thesaurus. Code C146753.

A disorder characterized by a state of generalized weakness with a pronounced inability to summon sufficient energy to accomplish daily activities. 